\title{
Heart Rate Variability Associated with Experienced Zen Meditation
}

\author{
M Hoshiyama $^{1,2}$, A Hoshiyama ${ }^{2}$ \\ ${ }^{1}$ The University of Tokyo, Tokyo, Japan \\ ${ }^{2}$ Meisei University, Tokyo, Japan
}

\begin{abstract}
To understand the control of hemodynamic events elicited by deep Zen meditation during Zazen, we studied heart rate in 5 experienced Zen meditators and 5 beginners. The study took place over 4 sets of Zen meditations in a quiet, Zen practice hall in Kamakura or Tokyo. Each set of Zazen lasted for 25 minutes which was preceded by specific respiratory exercise. The first sets were used for habituation, and the ECG data obtained from the following three sets were used for analysis. Power spectrum analysis showed distinctive change in frequency components. Low and high frequency components increased for experienced meditators. Most notably, detrended fluctuations analysis (DFA) of HRV were around $1 / 2$ for experienced meditators and 0.78 for beginners. We attribute the decrease of DFA exponent in experienced meditators to the effective regulation of mind during meditation toward the edge of sleep, but not quite over it.
\end{abstract}

\section{Introduction}

Zen is a traditional meditation method which utilizes unification of body and mind. Though Zen has been sophisticated highly in Japan, it is spreading widely into western world today, realizing deeper meditation with less effort.

While electroencephalogram (EEG) during Zen meditation has been studied in the past [1], there remains a lack of consensus whether heart rate during Zen practice elicit consistent heart rate variability (HRV) features. Research done in our laboratory aims at using the information contained in the fluctuation response of ECG heart rate to meditational state. To achieve this goal, it is necessary to understand better the mechanisms underlying the control of hemodynamic events through reciprocal autonomic activation elicited by meditational manipulation of body, respiration and mind. Specifically, we are interested in quantifying the degree of difference between ECG interbeat interval fluctuation responses seen during experienced Zen meditators and beginners.

\section{Methods}

We studied heart rate in 5 experienced Zen meditators who had been practicing several years of regular sitting Zen meditation (Zazen) and 5 beginners. The study took place over 4 sets of Zen meditations in a quiet, Zen practice hall in Kamakura or Tokyo. Each set of Zazen lasted for 25 minutes which was preceded by specific respiratory exercise. This respiratory exercise started with fully exhaling breath as one gently bent forward. Then one inhaled breath as gently rotating head backward, followed by exhaling breath as one gently rotated head forward.

Zazen prescribes a certain bodily posture. We sit on a cushion 5 to 15 centimeters thick that is placed on our blanket. We cross our legs so that one foot rests on the opposite thigh with the sole of our foot turned up and with our knees touching the blanket (lotus or half-lotus position). The torso should be kept straight, but it should not be strained. Except in the belly, there should be no strain anywhere. The head should be kept high with our eyes opened slightly and focused on a point about a meter away on the floor (Fig 1).

We should breath calmly and deeply through nose, but take 2 or 3 times as long to exhale as to inhale. We should also use lower abdominal muscles to accomplish Tanden breathing.

We should keep mind free from specific state of consciousness or distractions where 3 methods have been used for over a thousand years: concentration on breathing, so called shikantaza which means only to sit, and the koan. For beginners we use the first one by counting breaths mentally from 1 to 10 beginning with 1 again (susokukan), or by following the breaths, being aware only of inhalation when inhaling, and only of exhalation when exhaling (zuisokukan). For experienced meditator we use the latter two. In shikantaza we directly ignore any thoughts which might arise by simply letting any thoughts in and out without sticking to them. The koan is logically insoluble riddle, because it contains a contradiction. Logical solutions are rejected, and experienced meditators become 'one with koan'. 



Figure 1. Bodily posture of Zazen. Left: Statue of Buddha, Right: Schematic representation of lotus position. See text.

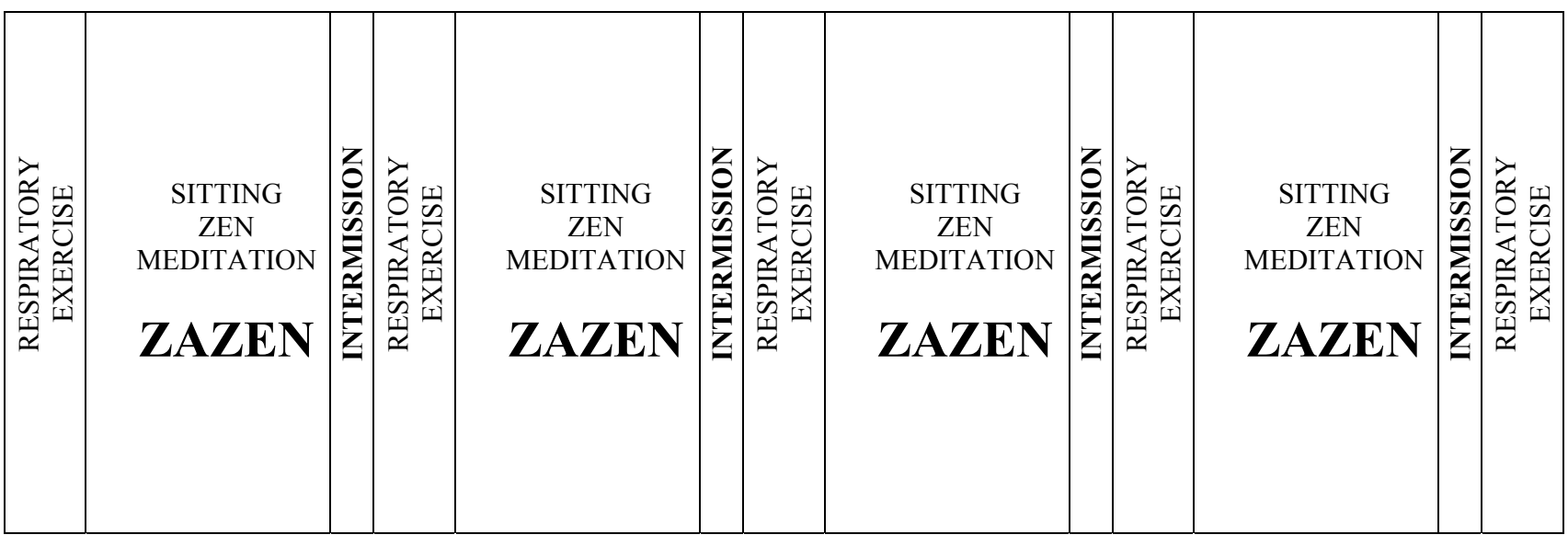

Figure 2. Sequence of events. 4 Zazen sessions were allocated with specific respiratory exercise before and with intermission after each sessions. The inter-beat intervals from ECG during last 3 Zazen meditations were used for analyses.

The first sets of Zazen were used for habituation, and the data obtained from the following three sets were used for analysis (Fig 2). The HR was obtained from ECG recordings.

We computed the detrended fluctuations analysis (DFA) exponents [2-5] of HRV for experienced meditators and beginners during Zazen. Exponents were calculated from linear fits to log-log plots of $F(n)$ versus $\mathrm{n}$ in the regime $70<\mathrm{n}<350$. We chose this fitting range to be above the regime of short-range correlations related

to breathing and below the $\mathrm{n}$ values where statistical errors become too large due to the finite length of each Zazen session.

\section{Results}

ECG data were analyzed for 10 subjects to test for autonomic states during each set of Zen meditation. Population averaged results for heart rate were not significantly different between experienced and 
beginners. Power spectrum analysis using Lomb method showed distinctive change in frequency components. Low frequency component (LF; $0.04 \mathrm{~Hz}$ to $0.15 \mathrm{~Hz}$ ) and high frequency component (HF; $0.15 \mathrm{~Hz}$ to $0.40 \mathrm{~Hz})$ increased for experienced meditators $(p=0.05)$. Very low frequency component $(\mathrm{VLF} ; 0.003 \mathrm{~Hz}$ to $0.04 \mathrm{~Hz}, \mathrm{p}=0.02$ ) and total frequency power $(\mathrm{TF} ; \mathrm{p}=0.05)$ increased relatively for beginners (Fig. 3).

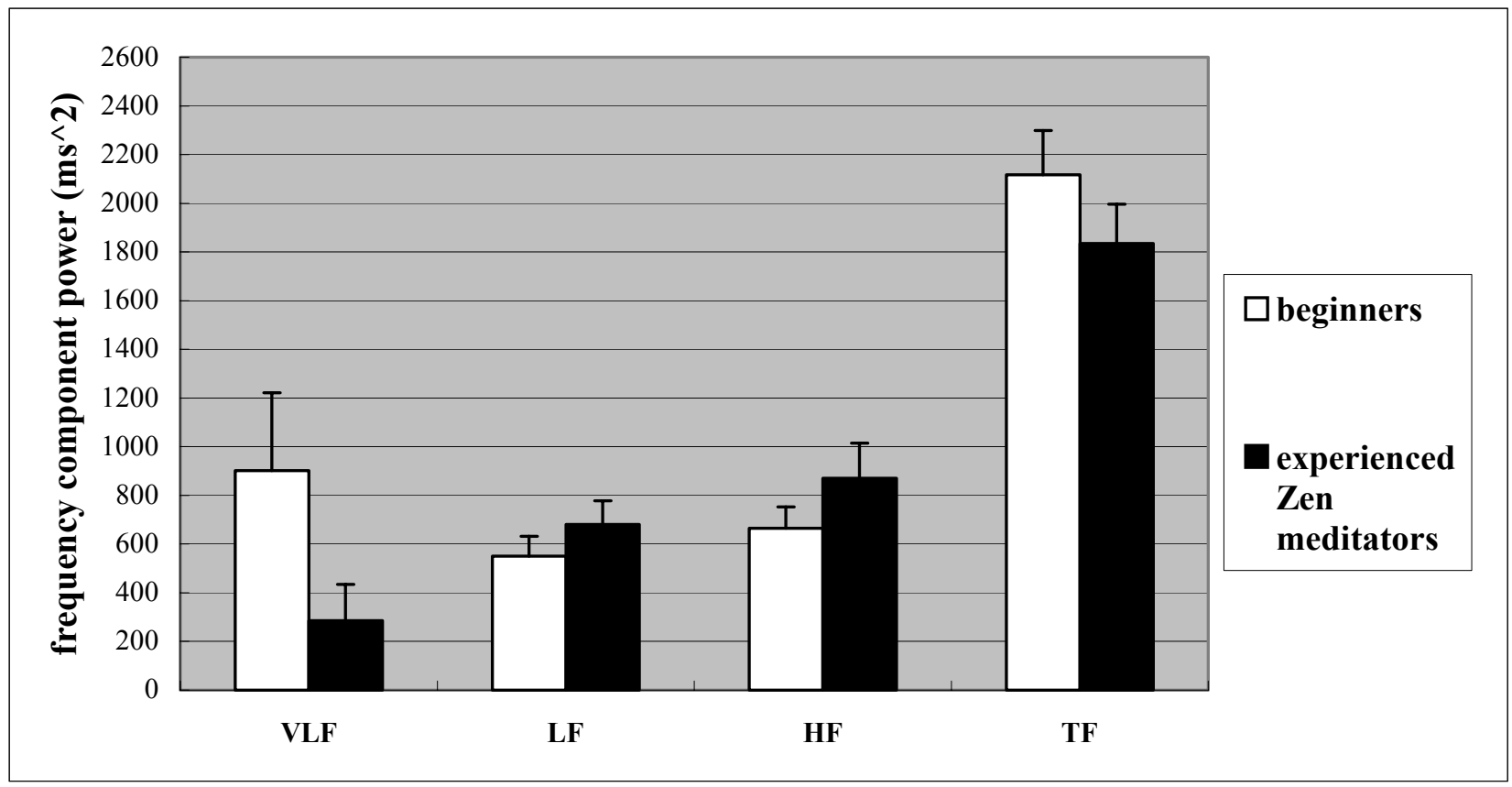

Figure 3. Frequency component power for VLF, LF, HF, TF of inter-beat intervals from ECG for beginners and experienced Zen meditators. Standard errors of the mean for 15 ECG segments per each group are presented as error bars.

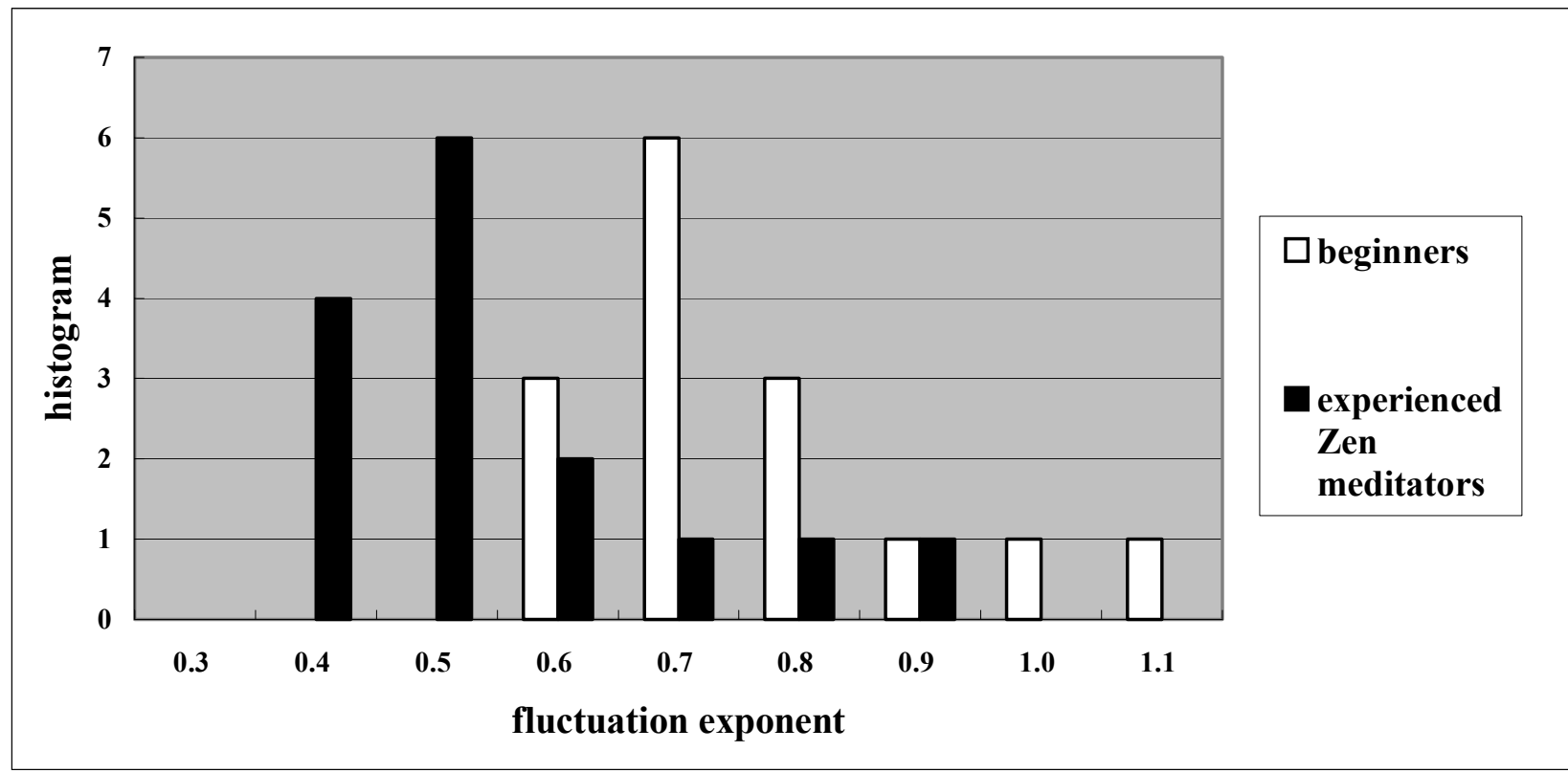

Figure 4. Histograms of DFA exponents of HRV for beginners and experienced Zen meditators. DFA exponents were around 0.78 for beginners and around 0.5 for experienced meditators. 
Most notably, population averaged results for DFA exponents of HRV differed between experienced meditators and beginners. The histograms were centered around $1 / 2$ for experienced meditators, showing almost no correlations. DFA exponents of HRV were around 0.78 for beginners, implicating long-range correlations (Fig. 4).

\section{Discussion and conclusions}

During Zen practice, we seat ourselves in a lotus posture, practice Tanden respiration, i.e., lower abdominal breathing, and keep mind free from specific state of consciousness. Deep meditaional state of Zen, namely Zanmai, can be accomplished after several years of Zen practice. This study was designed to elucidate the heart rate response to Zanmai by comparing beginners with experienced and practicing Zen meditators.

We could not find any difference in mean heart rate between beginners and experienced group. Significant differences exist, however, between the two groups in frequency components of the heart rate power spectrum. High frequency component is around the frequency of respiration, because it corresponds to respiratory sinus arrhythmia. Parasympathetic nervous system can transfer as high as $1 \mathrm{~Hz}$ where respiratory sinus arrhythmia is transferred, but sympathetic nervous system cannot transfer fluctuations higher than $0.15 \mathrm{~Hz}$. High frequency component is modulated by parasympathetic nervous system, but not by sympathetic nervous system [6,7]. Mayer wave [8] in arterial blood pressure reflect itself to heart rate through arterial baroreflex which generates low frequency component of the heart rate variability $[9,10]$. Recently this low frequency component has also been found in heart rate fluctuations under the artificial heart control suggesting central origin of this autonomic nervous rhythm [11]. Since the low frequency component is within the transferable frequency of sympathetic nervous system, this component is modulated by both sympathetic and parasympathetic nervous system. As mentioned above, low frequency component and high frequency component increased for the experienced group. We attribute the increase in high frequency component to the response of parasympathetic neural enhancement [12], and the increase in low frequency component to the response of both parasympathetic and sympathetic arousal mechanism during Zanmai state of deep Zen meditation.

In the previous sleep studies, DFA exponent of HRV decreased from 0.8 to around $1 / 2$ when they fall into light sleep from awake $[13,14]$. We attribute the decrease of DFA exponent in experienced meditators to the effective regulation of mind during highly sophisticated Zen meditation toward the edge of sleep, but not quite over it. This result suggests the possibility of HRV as a handy and quantitative evaluator for Zen meditation.

\section{Acknowledgements}

We are grateful to Zen masters Kadowaki and Nanrei for helpful suggestions on Zen practice, meditation and enlightenment.

\section{References}

[1] Kasamatsu A, Hirai T. An electroencephalographic study on the zen meditation (Zazen). Folia Psychiatr Neurol Jpn. 1966;20(4):315-36.

[2] Peng CK, et al. Quantification of scaling exponents and crossover phenomena in nonstationary heartbeat time series. Chaos 1995;5:82-87.

[3] Bak P, Tang C, Wiesenfeld K. Self-organized criticality. Phys Rev Lett 1987;59:381-384.

[4] Stanley HE. Introduction to Phase Transitions and Critical Phenomena. London:Oxford University Press, 1971.

[5] Goldberger AL. Non-linear dynamics for clinicians: chaos theory, fractals, and complexity at the bedside. Lancet 1996;347:1312-14.

[6] Akselrod S,Gordon D,Ubel FA,et al: Power spectrum analysis of heart rate fluctuation: A quantitative probe of beat-to-beat cardiovascular control. Sicence 1981;213:220222

[7] Pagani M,Lombardi F,Guzzetti S,et al: Power spetral analysis of heart rate and arterial pressure variabilities as a marker of sympatho-vagal interaction in man and conscious dog. Circ Res 1986;59:178-193

[8] Penaz J.: Mayer waves: History and methodology. Automedica 1978;2:135-141

[9] Madwed JB,Albrecht P,Mark RG,et al: Low-frequency oscillation in arterial pressure and heart rate: a simple computer model. Am J Physiol 1991;256:H1537-H1579

[10] Grasso R,Schena F,Gulli G,et al: Does low-frequency variability of heart period reflect a specific parasympathetic mechanism. J Auton Nerv Syst 1997:63:30-38

[11] Cooley RL,Montano N, Cogliati C,et al: Evidence for a central origin or the low-frequency oscillation in Rrinterval variability. Circulation 1998;98:556-561

[12] Hoshiyama M, Hoshiyama A. Heart Rate Variability Associated with the Influence of Mood. Computers in Cardiology 2005;32:583-586.

[13] Bunde A, Havlin S, Kantelhardt JW, Penzel T, Peter JH, Voigt K. Correlated and Uncorrelated Regions in HeartRate Fluctuations during Sleep. Phys Rev Lett 2000;85:3736-9.

[14] Hoshiyama M, Hoshiyama A. Heart Rate Variability Associated with Rapid Eye Movements during Sleep. Computers in Cardiology 2007;34:689-692.

Address for correspondence

Masaki Hoshiyama, M.D., Ph.D.

Omachi 1-9-12, Kamakura, Kanagawa, Japan

M.Hoshiyama@gmail.com 